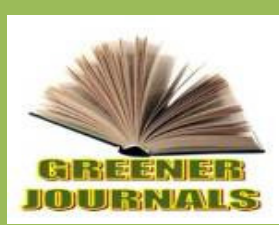

\title{
Genetic variability in Colletotrichum gloeosporioides (Penz.) isolated from cassava and yam from four agro- ecological zones of Ghana
}

\author{
${ }^{* 1}$ Appiah-Kubi Z., ${ }^{2}$ Apetorgbor A. K., '1Moses E., \\ ${ }^{1}$ Quian M., ${ }^{1}$ Thompson R., ${ }^{1}$ Appiah-Kubi D., ${ }^{1}$ Abrokwa L.
}

\author{
${ }^{1}$ CSIR-Crops Research Institute, P. O. Box 3785, Kumasi, Ghana \\ ${ }^{2}$ Department of Theoretical and Applied Biology, KNUST, Kumasi, Ghana
}

\section{ARTICLE INFO}

Article No.: 061715082

DOI: 10.15580/GJAS.2015.4.061715082

Submitted: 16/06/2015

Accepted: 20/06/2015

Published: 14/07/2015

${ }^{*}$ Corresponding Author

Appiah-Kubi Z.

E-mail:zipak1@yahoo.com

Keywords:

Anthracnose, Colletotrichum

gloeosporioides (Penz.), molecular

variations, ITS primers, RAPD
Anthracnose disease caused by Colletotrichum gloeosporioides (Penz.) Penz. and Sacc. is one of the most important fungal diseases of yam and cassava. To determine the genetic variations in $C$. gloeosporioides from the two crops, twenty-three isolates of the pathogen obtained from anthracnose-infected cassava stem and leaves and twenty-one isolates from yam leaves and vine from four agro-ecological zones of Ghana (Forest, Transition, Guinea and Coastal Savannah) were evaluated. Six species-specific internal transcribed spacer (ITS) primers were used in pairs to identify isolates to species. Amplification of the genomic DNA using specific primers ITS1 paired with ITS4 generated PCR products of band sizes between 500 to 600 bp which compared well with published data. Also eight Random Amplified Polymorphic DNA (RAPD) markers were used to determine genetic relationships among the $44 \mathrm{C}$. gloeosporioides isolates from cassava and yam. A phylogenetic tree constructed based on polymorphic bands generated with eight random primers, using the upgma method grouped forty-three of the isolates into two main clusters. The main clusters separated $C$. gloeosporioides isolates from cassava from those from yam. One isolate from cassava presented profiles completely different from the two main clusters and was grouped separately. 


\section{INTRODUCTION}

Colletotrichum gloeosporioides (Penz.), the causal organism of anthracnose disease is a facultative parasitic fungus belonging to the Melanconiales (order producing conidia in acervuli). The fungus produces hyaline, one-celled, and ovoid to oblong, slightly curved conidia, $10-15 \mu \mathrm{m}$ in length and $5-7 \mu \mathrm{m}$ in width. The waxy acervuli, which are produced in infected tissue, are sub-epidermal, and with setae (Padman and Janardhana, 2011; Kumar 2014). Identification of isolates in the $C$. gloeosporioides has traditionally been based on morphology, shape and size of conidia, presence or absence of setae and colony growth characteristics (Smith and Black, 1990; Sutton, 1992). However, these characteristics can be highly variable in C. gloeosporioides and differentiation based on these characteristics may be uncertain. Kumari et al. (2008) stated that pathogen variability is one of the main causes of failure to breed crop varieties or fungicidal control of plant diseases. Genetic variation of a pathogen is therefore a pre-requisite for any resistance breeding programme.

To overcome the limitations in traditional characterization, molecular markers can be used to bring about variations in isolates to the genetic level. Among the different molecular markers, there are some that are relatively economical, reliable and simple to use in assessing variations among plant pathogens. One such reliable molecular marker is the use of species-specific primers for fungus, the Internal Transcribed Spacers (ITS) primer to show the specificity of DNA from C. gloeosporioides isolates (White et al., 1990). To show genetic diversity, another economical and simple maker, the Random Amplification of Polymerized DNA (RAPD) can be employed. RAPD uses single primer of arbitrary nucleotide sequence to amplify genomic DNA to detect genetic polymorphism (Weising et al., 1995). The aim of this study was to characterize $C$. gloeosporioides isolates from cassava and yam from four agroecological zones of Ghana using molecular tools.

\section{MATERIALS AND METHOD}

\section{Single Spore Isolation of Pathogens}

Pieces of diseased tissues from leaves, stems and vines from cassava were obtained from four agroecological zones of Ghana. The pieces were surface sterilized by immersion in $5 \%$ sodium hypochlorite $(\mathrm{NaOCl})$ solution for $5 \mathrm{~min}$ followed by washing (three times) in sterile distilled water. They were then dried in a sterile Lamina flow hood, placed on PDA in Petri dishes and inoculated at $28 \pm 2^{\circ} \mathrm{C}$.

Mycelial growths from the plated tissues were transferred to fresh PDA in Petri dishes. They were then sub-cultured until pure cultures of $C$. gloeosporioides were obtained. These were maintained on PDA under Near Ultra Violet (NUV) light for one week.
Spore suspensions were prepared by adding $10 \mathrm{ml}$ sterile distilled water to the cultures followed by gentle surface agitation with a sterile inoculation needle. The spore suspensions were serially diluted from $1 \times 10^{-1}$ to $1 \times 10^{-5}$. From each of the $1 \times 10^{-4}$ and $1 \times 10^{-5}$ spore suspensions, $100 \mu \mathrm{l}$ were pipetted and spread onto the surface of freshly prepared water agar plates in 9-cm diameter Petri dishes and left for 24 hours. Sterile inoculation needles were used to gently pick germinating single conidia of $C$. gloeosporioides onto freshly prepared PDA plates. The plates were incubated under alternating cycles of NUV and darkness at $28^{\circ} \mathrm{C}$ for seven days. Single spore cultures produced were maintained on PDA.

\section{DNA Extraction}

Spore suspensions from forty-four $C$. gloeosporioides isolates from cassava and yam were produced from 10-day-old single spore cultures. Five hundred microlitres $(500 \mu \mathrm{l})$ of each spore suspension was used to inoculate $100 \mathrm{ml}$ of V8 Czapek Dox liquid medium. The inoculated medium was kept on an orbital shaker (Stuart Scientific), at $100 \mathrm{rpm}$ for 48 hours after which mycelia produced were harvested. Hundred milligrams $(100 \mathrm{mg})$ of mycelia from each isolate was frozen in liquid nitrogen and ground to powder. DNA was extracted from the powder using a modified protocol for DNA extraction from plant tissue using a commercial QIAGEN extraction kit (Qiagen sciences). The genomic DNA estimated was re-suspended in 50ul Tris-EDTA (10mM Tris-HCl [pH 8.0], 1mM EDTA and stored in a freezer until required (https://www.qiagen.com/resources/molecular-biologymethods/dna/).

\section{DNA Amplification with Specific Primers for Species Identification}

Six sets of universal internal transcribed spacer (ITS) primers (obtained from Sigma Aldrich, Germany) were used in pairs for their specificity to amplify the extracted genomic DNA. The ITS specific primers were: ITS1 (5'-CCGTAGGTGAACCTGCGG-3'), ITS1F (5'-CTTGGTCATTTAGAGGAAGTAA-3'), ITS4 (5'TCCTCCGCTTATT GATATGC-3'), ITS4-B (5'CAGGAGACTTGTACACGGTCCAG-3'), 5.8S (5'TCGATGAAGAACGC AGCG-3'), 5.8SR (5'-TCGATG AAGAA CGCA GCG-3'). Four primer pairing methods were used in the reactions; in the first one, ITS1 was paired with ITS4, in the second, ITS1 was paired with ITS1-F. ITS4 was paired with ITS4-B in the third pairing while $5.8 \mathrm{~S}$ was paired with $5.8 \mathrm{SR}$ for the last.

PCR was performed with a DNA thermocycler (Gen $A m p \AA$ PCR system 9700 version 3.09). Each PCR reaction mixture contained $2 \mu \mathrm{l}$ of genomic DNA, $25 \mathrm{mM} \mathrm{MgCl} 20.9 \mu \mathrm{l}, 10 \times$ PCR buffer $1 \mu \mathrm{l}, 10 \mathrm{mM}$ each dNTP $0.4 \mu \mathrm{l}, 0.05$ of Taq DNA polymerase (Biolab) and $0.25 \mu \mathrm{l}$ of each primer. The amplification cycle consisted of initial denaturation at $94^{\circ} \mathrm{C}$ for $5 \mathrm{~min}$ followed by 35 cycles consisting of $1 \mathrm{~min}$ at $94^{\circ} \mathrm{C}$, 2 min at $60^{\circ} \mathrm{C}$ and 2 -min at $72^{\circ} \mathrm{C}$ followed by final extension of $7 \mathrm{~min}$ at $72^{\circ} \mathrm{C}$. PCR products were 
separated in a $1.5 \%$ agarose gel, stained with ethidium bromide, viewed and photographed on a UV transilluminator (Stanley et al., 1996; Xiao et al., 2004).

\section{DNA Amplification with Random Primers}

Eight oligonucleotides: RP2-5'CCTGGGCTTG3'; RP45'CCTGGGCTGG3'; RP15- 5'CCTG GGTTTG3'; RP85 -5'GTGCTCGTGC3'; RP95 -5'GGGGGG TTGG3'; RP346 -5'AGGCGA ACG3'; RP3625'CCGCCTTACA3'; RP400- 5'GCCCTGATAT 3' were used for Randomized Amplification Polymorphic DNA (RAPD) analysis. PCR amplifications were carried out in $25 \mu \mathrm{l}$ mixtures containing $2 \mu \mathrm{l}$ of genomic DNA, $25 \mathrm{mM} \mathrm{MgCl} 20.9 \mu \mathrm{l}, 10 \times \mathrm{PCR}$ buffer $1 \mu \mathrm{l}, 10 \mathrm{mM}$ each dNTP $0.4 \mu \mathrm{l}, 0.05$ of Taq DNA polymerase (Biolab) and $0.5 \mu$ of primer. The reaction mixtures were incubated on a DNA thermocycler (Gen Amp® PCR system 9700 version 3.09). The PCR profile was of $3 \mathrm{~min}$ denaturation at $94^{\circ} \mathrm{C}$, followed by 35 cycles of $30 \mathrm{sec}$ at $94^{\circ} \mathrm{C}, 30 \mathrm{sec}$ at $45^{\circ} \mathrm{C}$ and $1 \mathrm{~min}$ at $72^{\circ} \mathrm{C}$, and then a 10 min extension at $72^{\circ} \mathrm{C}$. PCR products from the RAPD were separated in a $1.5 \%$ agarose gel, stained with ethidium bromide, viewed and photographed on a UV transilluminator (Xiao et al., 2004; Vinod and Benagi 2009). The multiple and single RAPD bands produced were scored manually and analyzed as binary data. The presence or absence of monomorphic and polymorphic bands was scored 1 and 0 respectively, and the results obtained for all primers were pooled in a single matrix for analysis.

\section{Analysis of Data}

The NTSYS PC software (Numerical Taxonomy System Applied Biostatistics, Inc.) (Rholf, 1994) was used for analysis of the genetic similarity of isolates based on Randomized Amplification Polymorphic DNA (RAPD) data. The data was analyzed as a binary matrix, from which a similarity matrix was built using the Darwin v5 - Jaccard coefficient (Jaccard, 1908). Data from the similarity matrix were used to produce dendrograms using the unweighted pair group method with arithmetic mean (UPGMA).

\section{RESULTS AND DISCUSSION}

\section{Species Identification with Specific Primers}

Of all the primer pairs used, only the ITS1 and ITS4 pair was able to amplify the genomic DNA from the Colletotrichum gloeosporioides isolates; no amplification product was observed with the ITS primer pairs ITS1/ITS1-F, ITS4/ITS4-B and 5.8s/5.8SR. Using the two universal primers, ITS1 and ITS4, a unique single band PCR product between 500-600bp was obtained from amplification of the ITS regions of the $C$. gloeosporioides isolates (Fig. 1). Out of the 44 isolates analysed, the specific primer ITS1 and ITS4 pair was able to generate amplification band patterns for 36 $(82 \%)$ of them. The pairing of primer ITS1 and ITS4 to amplify the genomic DNA samples to generate bands of fragment sizes between 500 and $600 \mathrm{bp}$ is in conformity with literature. For example Adaskaveg and Hartin (1997) paired primers ITS1 and ITS4 to generate $500 \mathrm{pb}$ fragment amplifications specific for $C$. gloeosporioides DNA. Vinnere et al. (2002) used ITS1, 5.8s and ITS2 primers to generate a single fragment of $600 \mathrm{bp}$ for $C$. gloeosporioides isolates. The close range of fragment sizes between 500 and $600 \mathrm{bp}$ generated between isolates from yam and those from cassava suggests that the isolates are closely related.

\section{PCR Amplification with Random Primers}

RAPD amplifications using the eight oligonuclotide primers yielded scorable bands for all the isolates of the fungal species. Primers RP2, RP4, RP95, RP15, RP85, RP346 and RP368 produced multiple bands (Fig. 2) while primer RP400 produced only a single band (Fig. 3).

Similarity coefficient values between $C$. gloeosporioides isolates from cassava and those from yam ranged from 0 to $39 \%$. There was $39 \%$ similarity between isolate C11a (isolate from cassava from forest ecological zone) and Y43b (isolate from yam from the coastal ecological zone) (Table 1). Similarity coefficient values among $C$. gloeosporioides isolates from the same crop ranged from 0 to $92 \%$ for $C$. gloeosporioides isolates from cassava (Table 2) and ranged from 0 to $58 \%$ for the isolates from yam (Table 3).

The dendrogram obtained from the cluster analysis of RAPD data portrayed two distinct clusters of the 48 C. gloeosporioides isolates from cassava and yam (Fig. 4). The first cluster (Cluster A) comprises 22 C. gloeosporioides isolates from cassava and three isolates from yam. The second cluster (Cluster B) consists of $18 \mathrm{C}$. gloeosporioides isolates, all from yam. Apart from the two major clusters in the dendogram there is also isolate C43b which is clearly distinguished from all the others. The genetic relatedness and diversity investigated through RAPD analysis separated C. gloeosporioides isolates from cassava from those of yam. The two clusters produced: Cluster $A$, comprising isolates mainly from cassava only and, Cluster B comprising isolates, all from yam is an indication that isolates from the two crops are genetically distinct. 
100bp Y11a Y11b Y12a Y12b Y21a Y21b Y22a Y22b Y23a Y31a Y31b Y32a Y32b Y33a Y33b Y41a Y41b 1Kb

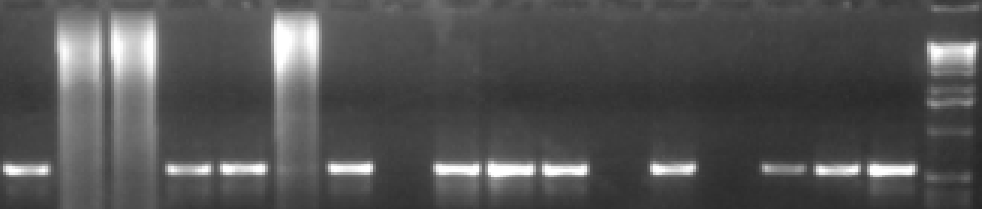

100bp Y42a Y42b Y43a Y43b C11a C11b C12a C12b C13a C13b C21a C21b C22a C22b C23a C23b C31a 1Kb

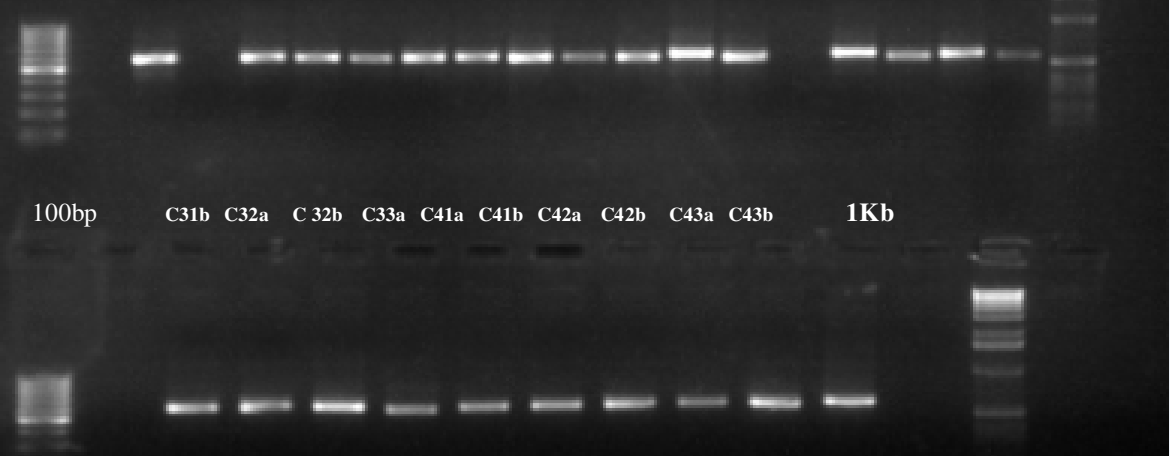

Fig. 1: PCR amplification bands from genomic DNA of Colletotrichum gloeosporioides using ITS1 and ITS4

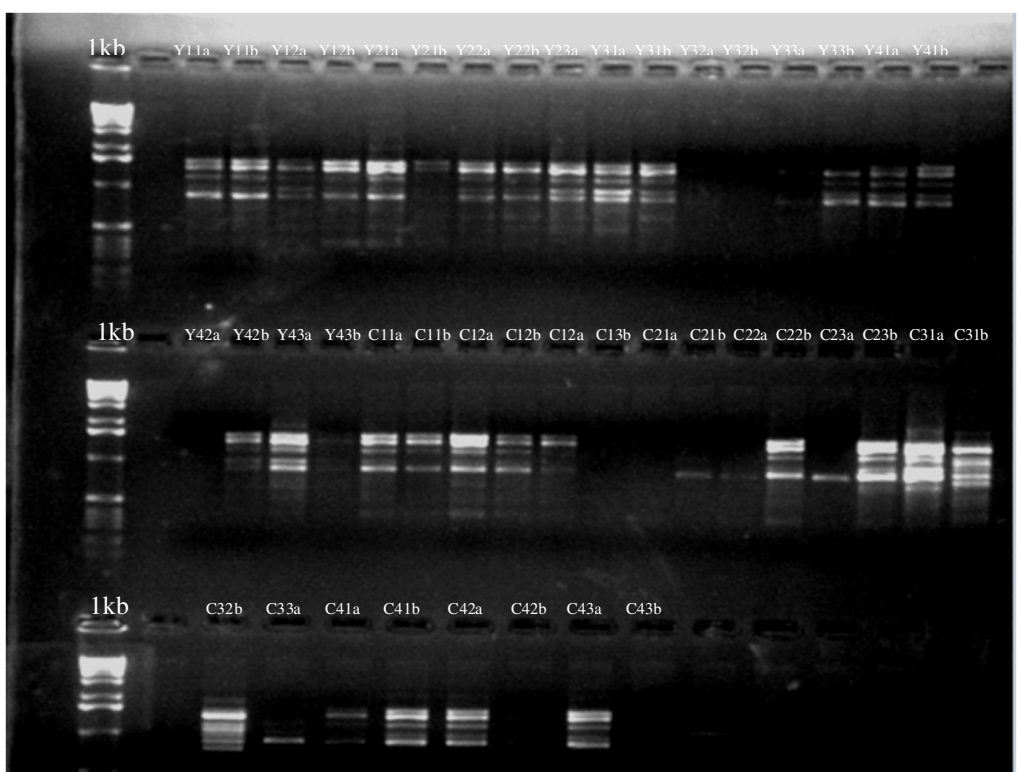

Fig. 2: RAPD profile of $C$. gloeosporioides generating multiple bands with primer RP4 


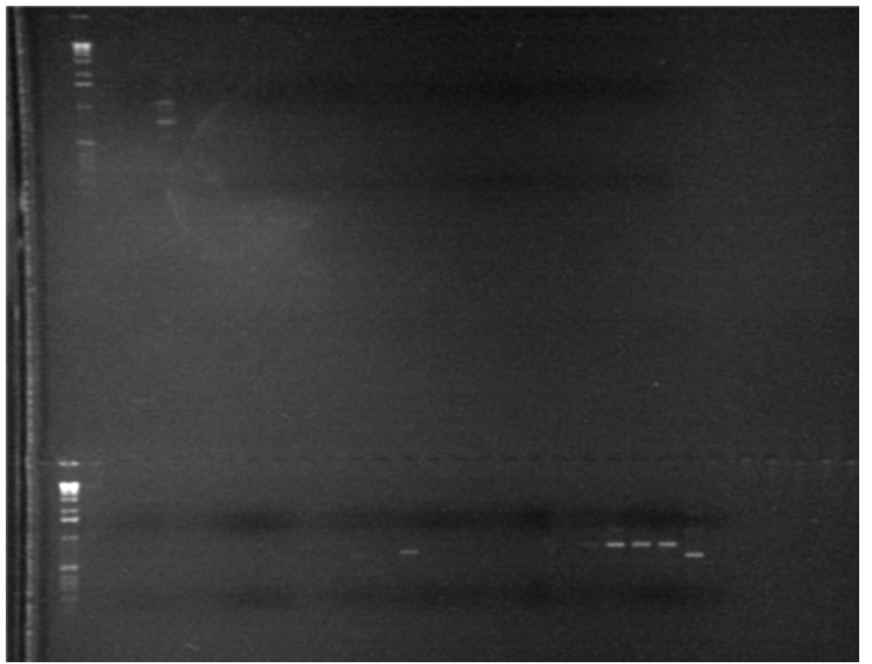

Fig. 3: RAPD profile of C. gloeosporioides generating a single band with primer RP400

Dendogram of Collectotrichum gloesporioides on Yam and Cassava

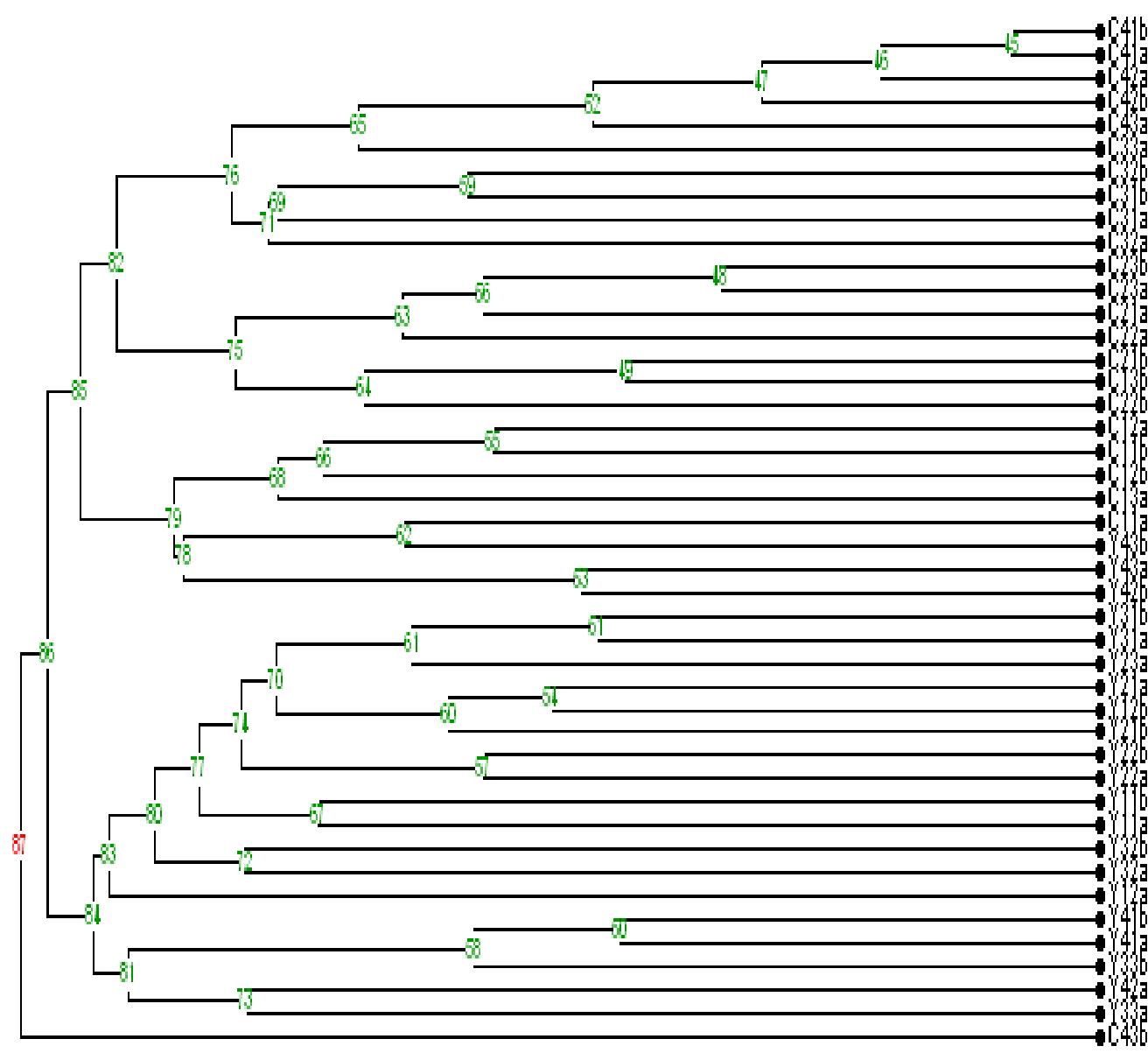

A

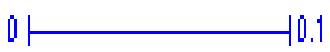

Fig. 4: Dendrogram showing genetic similarity between $C$. gloeosporioides isolates from yam and cassava based on RAPD profile 
Table 1: Similarity coefficient values of 44 C. gloeosporioides isolates from cassava and yam

\begin{tabular}{|c|c|c|c|c|c|c|c|c|c|c|c|c|c|c|c|c|c|c|c|c|c|}
\hline Unit & $\begin{array}{l}\text { Y11 } \\
a\end{array}$ & $\begin{array}{l}\text { Y11 } \\
\text { b }\end{array}$ & $\begin{array}{l}\text { Y12 } \\
a\end{array}$ & $\begin{array}{l}\text { Y12 } \\
\text { b }\end{array}$ & $\begin{array}{l}\text { Y21 } \\
a\end{array}$ & $\begin{array}{l}\text { Y21 } \\
\text { b }\end{array}$ & $\begin{array}{l}\text { Y22 } \\
a\end{array}$ & $\begin{array}{l}\text { Y22 } \\
\text { b }\end{array}$ & $\begin{array}{l}\text { Y23 } \\
a\end{array}$ & $\begin{array}{l}\text { Y31 } \\
a\end{array}$ & $\begin{array}{l}\text { Y31 } \\
\text { b }\end{array}$ & $\begin{array}{l}\text { Y32 } \\
a\end{array}$ & $\begin{array}{l}\text { Y32 } \\
\text { b }\end{array}$ & $\begin{array}{l}\text { Y33 } \\
\text { a }\end{array}$ & $\begin{array}{l}\text { Y33 } \\
\text { b }\end{array}$ & $\begin{array}{l}\text { Y41 } \\
a\end{array}$ & $\begin{array}{l}\text { Y41 } \\
\text { b }\end{array}$ & $\begin{array}{l}\text { Y42 } \\
a\end{array}$ & $\begin{array}{l}\text { Y42 } \\
\text { b }\end{array}$ & $\begin{array}{l}\text { Y43 } \\
a\end{array}$ & $\begin{array}{l}\text { Y43 } \\
\text { b }\end{array}$ \\
\hline $\begin{array}{l}\text { C11a } \\
\text { C11 }\end{array}$ & 0.15 & 0.13 & 0.11 & 0.19 & 0.21 & 0.10 & 0.19 & 0.15 & 0.08 & 0.06 & 0.10 & 0.00 & 0.06 & 0.07 & 0.12 & 0.08 & 0.11 & 0.14 & 0.25 & 0.25 & 0.39 \\
\hline b & 0.14 & 0.08 & 0.08 & 0.14 & 0.16 & 0.23 & 0.29 & 0.24 & 0.13 & 0.07 & 0.05 & 0.00 & 0.04 & 0.09 & 0.10 & 0.10 & 0.09 & 0.06 & 0.15 & 0.18 & 0.25 \\
\hline $\begin{array}{l}\text { C12a } \\
\text { C12 }\end{array}$ & 0.07 & 0.10 & 0.11 & 0.12 & 0.14 & 0.15 & 0.19 & 0.15 & 0.11 & 0.03 & 0.05 & 0.00 & 0.03 & 0.04 & 0.04 & 0.04 & 0.07 & 0.05 & 0.19 & 0.26 & 0.14 \\
\hline b & 0.29 & 0.19 & 0.09 & 0.19 & 0.21 & 0.15 & 0.23 & 0.16 & 0.15 & 0.11 & 0.11 & 0.00 & 0.05 & 0.17 & 0.06 & 0.10 & 0.13 & 0.03 & 0.13 & 0.15 & 0.11 \\
\hline $\begin{array}{l}\text { C13a } \\
\text { C13 }\end{array}$ & 0.19 & 0.11 & 0.08 & 0.14 & 0.21 & 0.08 & 0.17 & 0.08 & 0.09 & 0.07 & 0.11 & 0.00 & 0.00 & 0.09 & 0.05 & 0.05 & 0.09 & 0.06 & 0.25 & 0.25 & 0.11 \\
\hline b & 0.17 & 0.14 & 0.00 & 0.06 & 0.10 & 0.03 & 0.07 & 0.04 & 0.09 & 0.06 & 0.13 & 0.00 & 0.03 & 0.23 & 0.04 & 0.04 & 0.08 & 0.05 & 0.00 & 0.00 & 0.05 \\
\hline $\begin{array}{l}\text { C21a } \\
\text { C21 }\end{array}$ & 0.05 & 0.03 & 0.04 & 0.11 & 0.08 & 0.09 & 0.09 & 0.04 & 0.03 & 0.08 & 0.08 & 0.00 & .04 & 0.10 & 0.05 & 0.05 & 0.05 & 0.00 & 0.18 & 0.13 & 0.00 \\
\hline 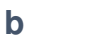 & 0.10 & 0.06 & 0.00 & 0.03 & 0.04 & 0.04 & 0.04 & 0.04 & 0.06 & 0.04 & 0.08 & 0.00 & 0.04 & 0.16 & 0.05 & 0.00 & 0.05 & 0.06 & 0.00 & 0.00 & 0.00 \\
\hline $\begin{array}{l}\text { C22a } \\
\text { C22 }\end{array}$ & 0.10 & 0.06 & 0.05 & 0.07 & 0.08 & 0.09 & 0.14 & 0.05 & 0.07 & 0.04 & 0.06 & 0.00 & .00 & 0.05 & 0.11 & 0.05 & 0.10 & 0.07 & 0.20 & 0.13 & 0.00 \\
\hline 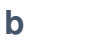 & 0.05 & 0.03 & 0.00 & .04 & 0.04 & 0.05 & 0.00 & 0.05 & 0.0 & 0.04 & 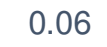 & 0 & & 2 & 0 & & 0 & 0 & & 0 & 0.00 \\
\hline $\begin{array}{l}\text { C23a } \\
\text { C23 }\end{array}$ & 0.05 & 0.03 & 0.04 & 0.07 & 0.04 & 0.04 & 0.04 & 0.00 & 0.00 & 0.04 & 0.03 & 0.00 & .04 & 0.05 & 0.00 & 0.00 & 0.00 & 0.00 & 0.08 & 0.06 & 0.00 \\
\hline 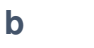 & 0.09 & 0.03 & 0.00 & 0.07 & 0.04 & 0.00 & 0.00 & 0.00 & 0.00 & 0. & & 0.00 & & 0 & 0.00 & 0.05 & 0.00 & 00 & & 0.00 & 0.12 \\
\hline $\begin{array}{l}31 a \\
31\end{array}$ & 0.15 & 0.12 & 0.00 & 0.03 & 0.08 & 0.09 & 0.09 & 0.09 & 0.06 & 0.04 & 0.05 & 0.00 & 0.04 & 0.10 & 0.11 & 0.00 & 0.05 & 0.06 & 0.00 & 0.00 & 0.06 \\
\hline 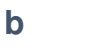 & 0.14 & 0.15 & 0.08 & 0.06 & 0.12 & 0.08 & 0.08 & 0.04 & 0.06 & 0.00 & & 0.00 & & 0.09 & 0.05 & 0.00 & 0.04 & 0.06 & & 0.11 & 0.05 \\
\hline $\begin{array}{l}32 a \\
32\end{array}$ & 0.17 & 0.20 & 0.04 & 0.13 & 0.10 & 0.11 & 0.20 & 0.12 & 0.12 & 0.06 & 0.10 & 0.00 & 0.03 & 0.13 & 0.08 & 0.08 & 0.12 & 0.00 & 0.06 & 0.10 & 0.10 \\
\hline b & 0.11 & 0.13 & 0.05 & 0.04 & 0.04 & 0.05 & 0.10 & 0.00 & 0.03 & 0.00 & & 0.00 & & 0.06 & 0.06 & 0.00 & 0.05 & 0.08 & & 0.15 & 0.07 \\
\hline C33a & 0.09 & 0.09 & 0.09 & 0.10 & 0.12 & 0.13 & 0.13 & 0.09 & 0.03 & 0.04 & 0.00 & 0.00 & 0.13 & 0.05 & 0.05 & 0.05 & 0.04 & 0.06 & 0.08 & 0.06 & 0.19 \\
\hline $\begin{array}{l}\text { C41a } \\
\text { C41 }\end{array}$ & 0.17 & 0.14 & 0.04 & 0.13 & 0.10 & 0.15 & 0.20 & 0.12 & 0.12 & 0.06 & 0.10 & 0.00 & 0.11 & 0.23 & 0.04 & 0.04 & 0.12 & 0.10 & 0.06 & 0.05 & 0.15 \\
\hline 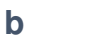 & 0.16 & 0.10 & 0.00 & 0.12 & 0.10 & 0.10 & 0.19 & (1.1 & 0.11 & 0.00 & 0.10 & 0.00 & 0.10 & 0.22 & 0.04 & 0.04 & 0.12 & 0.10 & & 0.04 & 0.14 \\
\hline $\mathrm{C} 42 \mathrm{a}$ & 0.13 & 0.14 & 0.04 & 0.09 & 0.11 & 0.16 & 0.16 & 0.12 & 0.09 & 0.07 & 0.10 & 0.00 & 0.07 & 0.18 & 0.04 & 0.00 & 0.04 & 0.05 & 0.06 & 0.05 & 0.10 \\
\hline $\mathbf{w}$ & 0.14 & 0.11 & 0.04 & 0.10 & 0.07 & 0.13 & 0.17 & 0.08 & 0.13 & 0.00 & 0.00 & 0.00 & 0.00 & 0.14 & 0.00 & 0.00 & 0.04 & 0.00 & 0.07 & 0.05 & 0.05 \\
\hline C43a & 0.10 & 0.06 & 0.04 & 0.07 & 0.04 & 0.04 & 0.09 & 0.00 & 0.03 & 0.04 & 0.05 & 0.00 & 0.04 & 0.10 & 0.00 & 0.00 & 0.10 & 0.06 & 0.08 & 0.06 & 0.00 \\
\hline & 0.11 & 0.06 & 0.00 & 0.04 & 0.09 & 0.05 & 0.10 & 0.05 & 0.03 & 0.09 & 0.03 & 0.00 & 0.05 & 0.00 & 0.06 & 0.00 & 0.00 & 0.00 & 0.00 & 0.00 & 0.07 \\
\hline
\end{tabular}


Table 2: Similarity coefficient values of 23 C. gloeosporioides isolates from cassava

\begin{tabular}{|c|c|c|c|c|c|c|c|c|c|c|c|c|c|c|c|c|c|c|c|c|c|c|c|}
\hline Unit & $\begin{array}{l}\text { C11 } \\
a\end{array}$ & $\begin{array}{l}\text { C11 } \\
b\end{array}$ & $\begin{array}{l}\text { C12 } \\
a\end{array}$ & $\begin{array}{l}\text { C12 } \\
\text { b }\end{array}$ & $\begin{array}{l}\text { C13 } \\
a\end{array}$ & $\begin{array}{l}\text { C13 } \\
\text { b }\end{array}$ & $\begin{array}{l}\text { C21 } \\
a\end{array}$ & $\begin{array}{l}\text { C21 } \\
\text { b }\end{array}$ & $\begin{array}{l}\text { C22 } \\
a\end{array}$ & $\begin{array}{l}\text { C22 } \\
\text { b }\end{array}$ & $\begin{array}{l}\text { C23 } \\
a\end{array}$ & $\begin{array}{l}\text { C23 } \\
b\end{array}$ & $\begin{array}{l}\text { C31 } \\
a\end{array}$ & $\begin{array}{l}\text { C31 } \\
\text { b }\end{array}$ & $\begin{array}{l}\text { C32 } \\
a\end{array}$ & $\begin{array}{l}\text { C32 } \\
\text { b }\end{array}$ & $\begin{array}{l}\text { C33 } \\
a\end{array}$ & $\begin{array}{l}\text { C41 } \\
a\end{array}$ & $\begin{array}{l}\text { C41 } \\
\text { b }\end{array}$ & $\begin{array}{l}\text { C42 } \\
a\end{array}$ & $\begin{array}{l}\text { C42 } \\
\text { b }\end{array}$ & $\begin{array}{l}\text { C43 } \\
a\end{array}$ & $\begin{array}{l}\text { C43 } \\
\text { b }\end{array}$ \\
\hline C11a & 1.00 & & & & & & & & & & & & & & & & & & & & & & \\
\hline C11b & 0.21 & 1.00 & & & & & & & & & & & & & & & & & & & & & \\
\hline $\mathrm{C} 12 \mathrm{a}$ & 0.17 & 0.47 & 1.00 & & & & & & & & & & & & & & & & & & & & \\
\hline C12b & 0.17 & 0.32 & 0.32 & 1.00 & & & & & & & & & & & & & & & & & & & \\
\hline C13a & 0.28 & 0.20 & 0.38 & 0.26 & 1.00 & & & & & & & & & & & & & & & & & & \\
\hline C13b & 0.04 & 0.00 & 0.04 & 0.28 & 0.24 & 1.00 & & & & & & & & & & & & & & & & & \\
\hline C21a & 0.11 & 0.14 & 0.11 & 0.17 & 0.23 & 0.27 & 1.00 & & & & & & & & & & & & & & & & \\
\hline $\mathrm{C} 21 \mathrm{~b}$ & 0.00 & 0.00 & 0.00 & 0.23 & 0.00 & 0.58 & 0.27 & 1.00 & & & & & & & & & & & & & & & \\
\hline $\mathrm{C} 22 \mathrm{a}$ & 0.18 & 0.15 & 0.12 & 0.08 & 0.15 & 0.13 & 0.44 & 0.18 & 1.00 & & & & & & & & & & & & & & \\
\hline $\mathrm{C} 22 \mathrm{~b}$ & 0.00 & 0.00 & 0.06 & 0.14 & 0.08 & 0.33 & 0.22 & 0.38 & 0.00 & 1.00 & & & & & & & & & & & & & \\
\hline $\mathrm{C} 23 \mathrm{a}$ & 0.11 & 0.07 & 0.11 & 0.13 & 0.14 & 0.27 & 0.56 & 0.27 & 0.44 & 0.38 & 1.00 & & & & & & & & & & & & \\
\hline $\mathrm{C} 23 \mathrm{~b}$ & 0.10 & 0.06 & 0.05 & 0.12 & 0.13 & 0.33 & 0.36 & 0.25 & 0.27 & 0.33 & 0.67 & 1.00 & & & & & & & & & & & \\
\hline C31a & 0.05 & 0.14 & 0.11 & 0.13 & 0.07 & 0.27 & 0.08 & 0.27 & 0.18 & 0.22 & 0.17 & 0.15 & 1.00 & & & & & & & & & & \\
\hline C31b & 0.10 & 0.13 & 0.16 & 0.12 & 0.06 & 0.11 & 0.14 & 0.14 & 0.36 & 0.08 & 0.23 & 0.13 & 0.33 & 1.00 & & & & & & & & & \\
\hline C32a & 0.13 & 0.17 & 0.14 & 0.28 & 0.05 & 0.14 & 0.06 & 0.12 & 0.06 & 0.07 & 0.06 & 0.00 & 0.27 & 0.31 & 1.00 & & & & & & & & \\
\hline C32b & 0.06 & 0.18 & 0.21 & 0.09 & 0.08 & 0.07 & 0.10 & 0.10 & 0.25 & 0.00 & 0.10 & 0.00 & 0.22 & 0.44 & 0.23 & 1.00 & & & & & & & \\
\hline C33a & 0.22 & 0.31 & 0.24 & 0.12 & 0.06 & 0.00 & 0.07 & 0.00 & 0.17 & 0.00 & 0.15 & 0.07 & 0.15 & 0.31 & 0.18 & 0.20 & 1.00 & & & & & & \\
\hline C41a & 0.18 & 0.24 & 0.14 & 0.19 & 0.11 & 0.09 & 0.19 & 0.06 & 0.20 & 0.07 & 0.19 & 0.11 & 0.19 & 0.31 & 0.33 & 0.14 & 0.43 & 1.00 & & & & & \\
\hline C41b & 0.17 & 0.22 & 0.18 & 0.18 & 0.16 & 0.14 & 0.18 & 0.05 & 0.19 & 0.13 & 0.25 & 0.17 & 0.25 & 0.29 & 0.32 & 0.13 & 0.40 & 0.92 & 1.00 & & & & \\
\hline $\mathrm{C} 42 \mathrm{a}$ & 0.14 & 0.25 & 0.20 & 0.15 & 0.18 & 0.15 & 0.20 & 0.06 & 0.21 & 0.15 & 0.29 & 0.19 & 0.29 & 0.33 & 0.28 & 0.15 & 0.36 & 0.77 & 0.85 & 1.00 & & & \\
\hline $\mathrm{C} 42 \mathrm{~b}$ & 0.10 & 0.20 & 0.16 & 0.16 & 0.13 & 0.11 & 0.23 & 0.07 & 0.25 & 0.08 & 0.23 & 0.13 & 0.14 & 0.38 & 0.31 & 0.18 & 0.31 & 0.75 & 0.69 & 0.67 & 1.00 & & \\
\hline $\mathrm{C} 43 \mathrm{a}$ & 0.11 & 0.14 & 0.11 & 0.13 & 0.14 & 0.06 & 0.27 & 0.00 & 0.30 & 0.00 & 0.27 & 0.15 & 0.08 & 0.33 & 0.19 & 0.22 & 0.25 & 0.58 & 0.54 & 0.50 & 0.60 & 1.00 & \\
\hline C43b & 0.06 & 0.08 & 0.00 & 0.14 & 0.08 & 0.14 & 0.00 & 0.10 & 0.00 & 0.00 & 0.00 & 0.00 & 0.22 & 0.08 & 0.23 & 0.00 & 0.09 & 0.07 & 0.06 & 0.07 & 0.00 & 0.00 & \\
\hline
\end{tabular}


Table 3: Similarity coefficient values of 21 C. gloeosporioides isolates from yam

\begin{tabular}{|c|c|c|c|c|c|c|c|c|c|c|c|c|c|c|c|c|c|c|c|c|c|}
\hline Unit & $\begin{array}{l}\text { Y11 } \\
a\end{array}$ & $\begin{array}{l}\text { Y11 } \\
\text { b }\end{array}$ & $\begin{array}{l}\text { Y12 } \\
a\end{array}$ & $\begin{array}{l}\text { Y12 } \\
\text { b }\end{array}$ & $\begin{array}{l}\text { Y21 } \\
a\end{array}$ & $\begin{array}{l}\text { Y21 } \\
\text { b }\end{array}$ & $\begin{array}{l}\text { Y22 } \\
a\end{array}$ & $\begin{array}{l}\text { Y22 } \\
\text { b }\end{array}$ & $\begin{array}{l}\text { Y23 } \\
a\end{array}$ & $\begin{array}{l}\text { Y31 } \\
a\end{array}$ & $\begin{array}{l}\text { Y31 } \\
\text { b }\end{array}$ & $\begin{array}{l}\text { Y32 } \\
\text { a }\end{array}$ & $\begin{array}{l}\text { Y32 } \\
\text { b }\end{array}$ & $\begin{array}{l}\text { Y33 } \\
\text { a }\end{array}$ & $\begin{array}{l}\text { Y33 } \\
\text { b }\end{array}$ & $\begin{array}{l}\text { Y41 } \\
a\end{array}$ & $\begin{array}{l}\text { Y41 } \\
\text { b }\end{array}$ & $\begin{array}{l}\text { Y42 } \\
a\end{array}$ & $\begin{array}{l}\text { Y42 } \\
\text { b }\end{array}$ & $\begin{array}{l}\text { Y43 } \\
a\end{array}$ & $\begin{array}{l}\text { Y43 } \\
\text { b }\end{array}$ \\
\hline $\begin{array}{l}\text { Y11a } \\
\text { Y11 }\end{array}$ & 1.00 & & & & & & & & & & & & & & & & & & & & \\
\hline b & 0.31 & 1.00 & & & & & & & & & & & & & & & & & & & \\
\hline $\begin{array}{l}\text { Y12a } \\
\text { Y12 }\end{array}$ & 0.14 & 0.27 & 1.00 & & & & & & & & & & & & & & & & & & \\
\hline b & 0.21 & 0.29 & 0.17 & 1.00 & & & & & & & & & & & & & & & & & \\
\hline $\begin{array}{l}\text { Y21a } \\
\text { Y21 }\end{array}$ & 0.29 & 0.25 & 0.23 & 0.52 & 1.00 & & & & & & & & & & & & & & & & \\
\hline b & 0.17 & 0.23 & 0.13 & 0.45 & 0.41 & 1.00 & & & & & & & & & & & & & & & \\
\hline $\begin{array}{l}\text { Y22a } \\
\text { Y22 }\end{array}$ & 0.21 & 0.17 & 0.13 & 0.17 & 0.23 & 0.29 & 1.00 & & & & & & & & & & & & & & \\
\hline b & 0.18 & 0.12 & 0.13 & 0.11 & 0.23 & 0.35 & 0.46 & 1.00 & & & & & & & & & & & & & \\
\hline Y23a & 0.20 & 0.27 & 0.10 & 0.25 & 0.31 & 0.38 & 0.42 & 0.34 & 1.00 & & & & & & & & & & & & \\
\hline $\begin{array}{l}\text { Y31a } \\
\text { Y31 }\end{array}$ & 0.12 & 0.21 & 0.06 & 0.25 & 0.28 & 0.22 & 0.11 & 0.23 & 0.31 & 1.00 & & & & & & & & & & & \\
\hline b & 0.14 & 0.27 & 0.04 & 0.27 & 0.24 & 0.28 & 0.22 & 0.26 & 0.49 & 0.56 & 1.00 & & & & & & & & & & \\
\hline $\begin{array}{l}\text { Y32a } \\
\text { Y32 }\end{array}$ & 0.00 & 0.18 & 0.06 & 0.18 & 0.13 & 0.21 & 0.03 & 0.10 & 0.20 & 0.28 & 0.30 & 1.00 & & & & & & & & & \\
\hline b & 0.06 & 0.26 & 0.09 & 0.23 & 0.18 & 0.23 & 0.06 & 0.03 & 0.15 & 0.29 & 0.31 & 0.25 & 1.00 & & & & & & & & \\
\hline $\begin{array}{l}\text { Y33a } \\
\text { Y33 }\end{array}$ & 0.15 & 0.15 & 0.03 & 0.08 & 0.03 & 0.10 & 0.10 & 0.10 & 0.14 & 0.06 & 0.24 & 0.11 & 0.17 & 1.00 & & & & & & & \\
\hline b & 0.15 & 0.13 & 0.00 & 0.12 & 0.13 & 0.10 & 0.19 & 0.15 & 0.14 & 0.21 & 0.21 & 0.07 & 0.14 & 0.12 & 1.00 & & & & & & \\
\hline $\begin{array}{l}\text { Y41a } \\
\text { Y41 }\end{array}$ & 0.11 & 0.13 & 0.07 & 0.09 & 0.10 & 0.07 & 0.10 & 0.11 & 0.14 & 0.17 & 0.18 & 0.07 & 0.22 & 0.16 & 0.40 & 1.00 & & & & & \\
\hline b & 0.19 & 0.12 & 0.03 & 0.11 & 0.09 & 0.06 & 0.21 & 0.18 & 0.24 & 0.19 & 0.26 & 0.03 & 0.17 & 0.24 & 0.50 & 0.58 & 1.00 & & & & \\
\hline $\begin{array}{l}\text { Y42a } \\
\text { Y42 }\end{array}$ & 0.04 & 0.05 & 0.04 & 0.00 & 0.00 & 0.00 & 0.08 & 0.08 & 0.06 & 0.11 & 0.14 & 0.08 & 0.21 & 0.25 & 0.04 & 0.14 & 0.18 & 1.00 & & & \\
\hline b & 0.10 & 0.06 & 0.15 & 0.11 & 0.18 & 0.09 & 0.14 & 0.10 & 0.07 & 0.08 & 0.06 & 0.00 & 0.04 & 0.00 & 0.05 & 0.11 & 0.05 & 0.14 & 1.00 & & \\
\hline $\begin{array}{l}\text { Y43a } \\
\text { Y43 }\end{array}$ & 0.08 & 0.08 & 0.12 & 0.09 & 0.15 & 0.07 & 0.21 & 0.17 & 0.09 & 0.07 & 0.08 & 0.00 & 0.03 & 0.04 & 0.14 & 0.19 & 0.17 & 0.17 & 0.55 & 1.00 & \\
\hline & 0.13 & 0.08 & 0.00 & 0.06 & 0.07 & 0.07 & 0.12 & 0.12 & 0.09 & 0.03 & 0.05 & 0.00 & 0.07 & 0.13 & 0.04 & 0.09 & 0.08 & 0.17 & 0.06 & 0.22 & 1.00 \\
\hline
\end{tabular}




\section{CONCLUSION}

The use of ITS primers and Random Amplified Polymorphic DNA (RAPD) molecular markers were able to detect the variations among $C$. gloeosporioides isolates from cassava and those from yam. The pairing of ITS primers (ITS1 and ITS4) to generate PCR products of band sizes between 500 to $600 \mathrm{bp}$ made the pair species-specific primers for the amplification of C. gloeosporioides isolates. The use of RAPD analysis with simple primers had generated results that are reliable. The two major groups produced by the dendrogram obtained from the cluster analysis of RAPD data is an indication that $C$. gloeosporioides isolates from cassava is genetically not the same as the C. gloeosporioides isolates from yam.

Isolate C43b stood out as a genetically distinct lineage from all the others. Further molecular analyses using other genes and more sensitive approaches are however required for sequence analyses of the pathogen.

\section{REFERENCES}

Adaskaveg JE and Hartin RJ (1997). Characterization of Colletotrichum acutatum isolates causing anthracnose of almond and peach in California. Plant Pathol. 87(9):979 - 987.

Jaccard P (1908). Nouvelles recherches sur la distribution florale. Bul. Soc. Vaudoise Sci.

Nat. 44:223-270.

Kumar GA (2014). Colletotrichum gloeosporioides: Biology, Pathogenicity and Management in India. Journal of Plant Physiology \& Pathology 2(2):1-11. http://dx.doi.org/10.4172/2329-955X.1000125

Kumari SS, Adiver VI, Benagi AS and Nadaf $\mathrm{HL}$ (2008). Molecular variation in Phaeoisariopsis personata (Berk. and M.A. Curtis) van Arx causing late leaf spot of groundnut (Arachis hypogea L.). Karnataka J. Agric. Sci. 22(2): 336-339.

Padman M and Janardhana GR (2011). Occurrence and characterization of Colletotrichum gloeosporioides isolated from Murraya koenigii. New York Science Journal. 4(8): 70-76
Smith BJ and Black LL (1990). Morphological, cultural, and pathogenic variation among Colletotrichum species isolated from strawberry. Plant Dis. 74:6976.

Stanley F, Talma K and Ezra S (1996). Characterization of Colletotrichum gloeosporioides isolates from avocado and almond fruits with molecular and pathogenicity tests. Applied and Environmental Microbiology 6:1014-1020.

Sutton BC (1992). The genus Glomerella and its anamorph Colletotrichum. In: Colletotrichum: Biology, Pathology and Control. J.A. Bailey and M.J. Jeger (eds). CAB International, Wallingford, UK: $1-26$.

Rholf FJ (1994). NTSYS-pc, numerical taxonomy and multivariate analysis system. Exeter Software, Applied Biostatistics, N.Y. Saharan Africa. pp 432439.

Vinnere O, Fatehi J, Wright SAl and Gerhardson B (2002). The causal agent of anthracnose of Rhododendron in Sweden and Latvia. Mycol. Res. 106:60 - 69.

Vinod T and Benagi VI (2009). Studies on the cultural and nutritional characteristics of Colletotrichum gloeosporioides, the causal organism of papaya anthracnose. Karnataka J. Agric. Sci. 22 (4):787789.

Weising K, Nybon M, Wolf K and Meyer W (1995). DNA finger printing in plants and fungi. PI. Gen. Res. Newsltr. 97:3-39.

White T J, Bruns T, Lee S and Taylor J (1990). Amplification and direct sequencing of fungal ribosomal RNA genes for phylogenetics. In: PCR Protocols. Innis, M. A., Gelfand, D. H., Sninsky, J. H., White, T. J. (eds). Academic Press, San Diego, USA pp 315-322.

Xiao CL, Mackenzie SJ and Legard DE (2004). Genetic and pathogenic analyses of Colletotrichum gloeosporioides isolates from strawberry and non-cultivated hosts. Phytopathology 94: 446-53.

https://www.qiagen.com/resources/molecular-biologymethods/dna/. DNA Protocols and Applications$\begin{array}{lll}\text { Qiagen. } & \text { Assessed } & 11 / 05 / 15\end{array}$ 